\title{
Joint international efforts to combat a still-deadly disease: the next generation
}

Hubert J. STEIN

Department of Surgery, Klinikum Nuernberg, Nuremberg, Germany

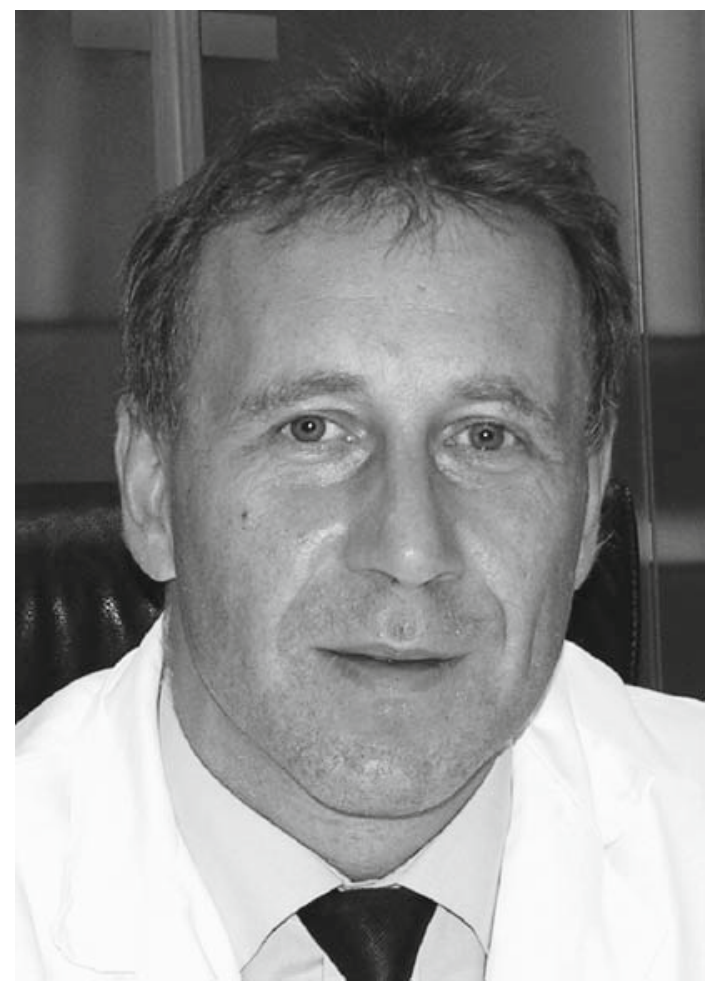

Hubert J. Stein, M.D., F.A.C.S.

Department of Surgery, Klinikum Nuernberg, Nuremberg

Despite an overall declining incidence, gastric cancer remains a prevalent and for many patients still-deadly disease in the Western world. A great deal has been learned from our colleagues in the Eastern world and from scientific studies performed in the West during the past years. Much progress has been made in terms of early diagnosis, pathogenesis, tumor biology, minimally invasive and endoscopic treatment, standardization of surgical approaches and procedures, perioperative management, and pre-/postoperative treatment. Prevention, molecular markers, epigenetics, multidisciplinary approaches, and individualized/tailored therapy are the issues of today.

But many more challenges lie ahead. At least in the Western world, the face of gastric cancer is changing. Here, we are still confronting a largely unexplained and rising incidence of proximal gastric cancer and cancer of the esophagogastric junction. The problem of peritoneal spread in the absence of distant organ metastases is still unresolved. Very little progress is being made in combating systemic disease.

Despite many similarities of gastric cancer between the East and the West, a direct transfer of knowledge and concepts is not always possible. However, an international journal focusing on one disease, as we have here with the journal Gastric Cancer, provides a unique opportunity for communication and a combined effort to combat this disease

I am glad and grateful to have the chance to continue the East-West collaboration together with my dear friend Yuko Kitagawa as an editor of the journal very much in the spirit that has been created by our predecessors Takeshi Sano and Jörg Rüdiger Siewert.

Hubert J. Stein Editor-in-Chief 